\title{
Muerte, estética y filosofía: el arte de filosofar según Schopenhauer
}

\author{
Death, aesthetics and philosophy: the art of philosophizing according to \\ Schopenhauer
}

\author{
Roger A. Pérez García \\ Pontificia Universidad Católica del Perú (PUCP) \\ E-mail:roger.perezg@pucp.edu.pe
}

Sumilla: Proponemos interpretar el problema del miedo a la muerte en la filosofía de Schopenhauer como un error basado en la ilusión trascendental. En tanto error, este miedo es erradicado por argumentos, pero, en tanto ilusión, Schopenhauer debe proveernos de una vía para corregir la intuición. Esta vía, sostendremos, es la intuición estética. En ese sentido, analizamos las facultades artísticas del genio y su relación con el quehacer del filósofo; en particular, la contemplación estética como captación intuitiva de las Ideas platónicas. Sostendremos que las Ideas funcionan como bisagra entre el mundo como representación y el mundo como voluntad. Estas Ideas permiten al filósofo 1) vencer la resistencia interna que el sujeto opone a la tesis idealista al proveerla de contenido intuitivo, y 2) romper con la ilusión trascendental, al revelar el carácter ilusorio del mundo empírico e interpretarlo en función de distintos grados de objetivación de la Voluntad.

Palavras-chave: Muerte; Ilusión; Estética; Filosofía; Filosofar.
Abstract: The present paper proposes to read the problem of the fear of death in Schopenhauer's philosophy as a mistake based in the transcendental illusion. As a mistake, this fear can be eradicated by arguments, but, as an illusion, Schopenhauer must supply us of a way to correct the intuition. This way, we hold, is the aesthetic intuition. Accordingly, we analyze the artistic faculties of the genius and its relation with the work of the philosopher; particularly, the aesthetic contemplation as the intuittive reception of the platonic Ideas. We will argue that the Ideas work as a hinge between the world as representation and the world as will. The Ideas allow the philosopher to 1) overcome the inner resistance that the subject opposes to the idealistic thesis by providing the thesis with intuitive content, and 2) to break the transcendental illusion by revealing the illusory character of the empirical world and interpret it according to the different levels of objectification of the thing in itself, the will.

Keywords: Death; Illusion; Aesthetics; Philosophy; Philosophizing. 
$\mathrm{E}$ n el hombre, afirma Schopenhauer, la conciencia y la voluntad se han separado de tal manera que "al encontrase juntos puedan asombrarse el uno del otro"1. Esta separación produce aquello que para Schopenhauer es característico del hombre: el miedo a la muerte. Ante este miedo, el hombre cae en cuenta de "la finitud de la existencia" y de "la vanidad de todo esfuerzo"2. Esta situación define al hombre como animal metaphysycum, pues le permite "asombrarse de lo habitual y cotidiano"3. Así, el asombro que conduce a la filosofía y del que surge la metafísica es, también, el que da pie al planteamiento de todas las preguntas filosóficas, puesto que el "asombro ante lo cotidiano" es el que "induce a convertir la totalidad del fenómeno en un problema" (tarea teórica) y, también, a la "consideración del sufrimiento y la necesidad de la vida" (tarea práctica de la filosofía) ${ }^{4}$.

En vista de esto, es importante preguntarnos por el origen del temor a la muerte del cual surge la metafísica. Encontramos que el miedo a la muerte se funda en el egoísmo del individuo como una afirmación de su vida o voluntad individual ${ }^{5}$. Esto es paradójico si se considera que la afirmación de la voluntad de vivir es también la que esquiva el miedo a la muerte, pues "a quien le satisfaga la vida tal como es, el que la afirme de cualquier manera, podrá confiadamente considerarla infinita y conjurar el miedo a la muerte como un engaño que le infunde el disparatado temor de quedar alguna vez privado del presente y le crea la ilusión de un tiempo sin presente"6.

Estamos, así, frente a una paradoja. Por un lado, el hombre teme a la muerte no en la parte cognoscente de su ser, sino en tanto objetivación de la voluntad ciega de la que está llena todo lo viviente ${ }^{7}$.Por otro lado, y al mismo tiempo, la voluntad de vivir tiene asegurada la vida y mientras estamos llenos de voluntad de vivir no podemos estar preocupados por nuestra existencia ni siquiera ante la visión de la muerte ${ }^{8}$.

Así, al considerar el miedo a la muerte, no solo hacemos frente a la distinción entre el ser cognoscente y ser volitivo, o entre el fenómeno y la cosa en sí; sino que hacemos frente a una determinada configuración de la voluntad. Esta configuración es la voluntad objetivada en el individuo. Schopenhauer radicalizará esta postura y, al igualar

${ }^{1}$ SCHOPENHAUER, A.,MVR II, cáp. 17, p. 198.

${ }^{2} C f$. SCHOPENHAUER, A., MVR II, cáp. 17, p. 199.

3 Ibidem.

${ }^{4}$ Cf. Ibidem.

${ }^{5}$ Cf. SCHOPENHAUER, A., MVR I, §54, p. 338-339.

${ }^{6}$ SCHOPENHAUER, A., MVR I, §54, p. 336.

${ }^{7}$ Cf. SCHOPENHAUER, A., MVR I, §54, p. 331-332.

${ }^{8}$ Cf. SCHOPENHAUER, A., MVR I, §54, p. 331.

Muerte, estética y filosofía: el arte de filosofar según Schopenhauer 
voluntad individual y organismo afirmará que "lo que nos resulta más temible de la muerte no es el término de la vida (...) sino más bien la destrucción del organismo: porque este es la voluntad misma que se presenta como cuerpo" 9 . Por tanto, el interés en preservar la vida fenoménica y el temor ante la muerte sirven a los intereses de la voluntad individual. La mera separación entre el ser racional y el ser volitivo del hombre, entre razón y voluntad, es solo la forma fenoménica en la que el hombre se vuelve consciente y temeroso ante su muerte. En el fondo, la resistencia y el temor a la muerte se fundan en una dialéctica de la voluntad consigo misma, en la que, como afirma Jacquette, la mente no puede adecuadamente comprender el concepto de muerte, puesto que todos sus conceptos se derivan de la objetivación de la voluntad. Por ello, la mente trata fallidamente de representar la muerte como la negación de su conocimiento y experiencia de la vida, como un olvido de la conciencia en el que la voluntad en su conjunto deja de existir ${ }^{10}$.

Ante este panorama, Schopenhauer considera que la muerte es "el verdadero genio inspirador o el musageta de la filosofía"11. Si reparamos en lo expuesto hasta el momento, la confrontación del hombre con la muerte (el esfuerzo por representar lo irrepresentable) es, en cierto sentido, análoga a la confrontación con lo inexplicable. Ambas se fundan en el encuentro con el límite de la representación. En la misma analogía, la tesis del carácter representacional del mundo (tesis idealista) debería ser comprendida como ese encuentro con los límites del mundo representacional del cual debe partir la filosofía.

Tenemos, así, el enigma teórico y el enigma moral que competen a la filosofía. Sin embrago, la filosofía es, para Schopenhauer, un único pensamiento cuya unidad, sostenemos, puede ser expuesta en la unidad metodológica con la que Schopenhauer pretende dar respuesta al enigma teórico y moral del mundo. Hemos de preguntarnos, por ello, si el problema moral del miedo a la muerte nos conduce a consideraciones de orden teórico y a la pregunta por cómo ha de proceder la filosofía si su punto de inicio es el límite de la representación. Comencemos por reafirmar la problemática de la explicación del miedo a la muerte:

\footnotetext{
${ }^{9}$ SCHOPENHAUER, A., MVR II, cáp. 41, p. 521.

${ }^{10}$ JACQUETTE, D. "Schopenhauer on Death". En: The Cambridge Companion to Schopenhauer, editado por Christopher Janaway, p. 297.

11 SCHOPENHAUER, A., MVR II, cap. 41, p. 515.

Muerte, estética y filosofía: el arte de filosofar según Schopenhauer
} 
La vida no es algo bueno sino sufrimiento, caducidad, camino continuo hacia la muerte. Todo lo que podemos es prorrogar el momento definitivo de morir. Vista de una manera más metafísica, la muerte es parte del destino de los individuos que nacen y perecen mientras que la especie, la vida como tal, la naturaleza en su totalidad, permanece. De ahí surge el problema de la explicación del miedo de la muerte. Por una parte, el hombre es el único ser que sabe que morirá, mientras que los animales viven sin la conciencia de la muerte. Por otra parte, no sólo los hombres sino también los animales temen la muerte cuando se presenta como inminente. Este hecho demuestra que el miedo de la muerte no depende de ese conocimiento de la muerte que falta a los animales, sino que tiene su origen en la naturaleza misma. En el miedo de la muerte se expresa la voluntad de vivir que se opone a la extinción de la vida ${ }^{12}$.

Esta cita nos muestra que al considerar el miedo a la muerte debemos abordar el problema en sus distintos niveles: desde el miedo y la angustia real del individuo, pasando por la vista panorámica de la metafísica sobre la naturaleza y la vida de la especie hasta el reconocimiento final de la dialéctica de la Voluntad consigo misma.

En consonancia con la filosofía de Schopenhauer, las gradaciones presentadas aquí no transforman el objeto, sino que lo explican desde un punto de vista cada vez más elevado. No se "desrealiza" la muerte, si por tal se entiende que esta no ocupe un lugar dentro de ningún ámbito de la realidad, sino que se analiza la experiencia a fin de comprender las condiciones que la hacen posible. De ahí que, lo realmente paradójico sea que esas mismas condiciones contengan ya la clave de su superación. En ese sentido, el temor a la muerte y la muerte misma no son confrontadas a un esquema metafísico idealizado de cómo el mundo debería ser. Puesto que, para Schopenhauer, "toda filosofía es siempre teórica, ya que le es esencial, sea cual sea el objeto inmediato de la investigación, actuar siempre de forma puramente contemplativa e investigar, no prescribir (stets rein betrachtend zu verhalten und zu forschen, nicht vorzuschreiben)"13. No es de sorprender, entonces, que Schopenhauer abra el cuarto libro de su obra dedicado a la consideración moral del mundo reafirmando el carácter teórico de la filosofía y refiriéndose a la definición de filosofía como un "interpretar y explicar lo existente, llenar el ser del mundo que se expresa a cada cual de manera comprensible in concreto, es decir, como sentimiento, a un claro conocimiento abstracto de la razón, y eso en todos los sentidos posibles y desde cualquier punto de vista"14.

${ }^{12}$ SCHÖNDORF, H. "La muerte en la filosofía de Arthur Schopenhauer". En: Revista Portuguesa de Filosofía. Tomo 65: O Dom, a Verdade, e a Morte: Abordagens e Perspectivas, p. 1204.

13 SCHOPENHAUER, A., MVR I, §53, p. 327.

14 Ibidem.

Muerte, estética y filosofía: el arte de filosofar según Schopenhauer 
Cabe preguntarnos entonces qué forma de proceder conduce a la filosofía desde la angustia ante la muerte, la finitud y el dolor, hasta la comprensión de que "nuestro apego de la vida es [...] insensato y ciego y se puede explicar solamente por el hecho de que nuestra esencia es una voluntad de vivir desconocedora y ciega. Es decir, que en el miedo de la muerte se expresa la esencia originaria de nuestro ser, pero si el conocimiento vence, el hombre podrá acercarse a la muerte con ánimo y calma"15. La promesa de la filosofía está, así, contenida en su propio proceder a modo tanto de una expansión o elevación del conocimiento, como de una supresión de la angustia a través precisamente de dicho conocimiento. Así, no es posible disociar el aspecto metodológico que subyace al desarrollo de la filosofía de Schopenhauer de la necesidad metafísica propia del ser humano. El proceder de la filosofía debe hacer frente a esta necesidad, especialmente allí donde esta necesidad se muestra más apremiante, en el conocimiento de la propia finitud. La consideración sobre la filosofía y la muerte se encuentra en el capítulo 17 del segundo volumen que complementa el §15. Allí, Schopenhauer presenta una revisión general de las definiciones y conceptos de los que se sirve la filosofía. Uno de ellos es el concepto de ilusión (der Schein). Esta tiene su origen en un defecto de la naturaleza misma del entendimiento. A partir de la sensación el entendimiento construye la representación empírica que, de acuerdo con las condiciones a priori del conocimiento (en particular el principio de razón suficiente), se presenta como una realidad externa e independiente del sujeto. Este emplazamiento de realidad empírica (Wirklichkeit) es considerada por Schopenhauer "una inferencia del entendimiento del efecto a la causa (einerVerstandesschlu $\beta$ von der Wirkungauf die Ursache)" que, en tanto "inferencia de lo fundamentado al fundamento (vomBegründetenauf den Grund der Schluß)", no es del todo segura. Esta ausencia de certeza establece, para Schopenhauer, la posibilidad de "la falsa apariencia o falsa ilusión (der falscheSchein) como engaño de los sentidos"16. Schopenhauer terminará por considerar que la posibilidad de la ilusión es atribuible a todo conocimiento empírico y, consiguientemente, a toda la ciencia natural ${ }^{17}$.

En sentido estricto, toda la realidad fenomenal cae bajo la posibilidad de la ilusión, debido a la naturaleza misma del entendimiento. La falsa ilusión, por el contrario, hace referencia a fenómenos anormales de la fisiología como la visión y el

${ }^{15}$ SCHÖNDORF, H., op. cit., 1196. El sombreado es mío

${ }^{16} \mathrm{C}$. SCHOPENHAUER, A., MVR I, §15, p. 128.

${ }^{17}$ Cf.Ibidem.

Muerte, estética y filosofía: el arte de filosofar según Schopenhauer 
tacto doble ${ }^{18}$. Se abre camino, pues, para considerar la realidad fenoménica (Wirklichkeit) como una forma de ilusión. Tal parece ser el caso cuando Schopenhauer afirma que, en vista de la separación entre fenómeno y cosa en sí, "este mundo que aparece a los sentidos no tiene un verdadero ser sino un incesante devenir, es y al mismo tiempo no es, y su captación no es tanto un conocimiento como una ilusión (Schein) ${ }^{19 ”}$. Al mismo tiempo, sin embargo, en el $\S 5$, Schopenhauer afirma que, a pesar de ser solo representación, el mundo "no (...) es engaño ni ilusión (Schein): se da como lo que es, como representación y, por cierto, como una serie de representaciones cuyo nexo común es el principio de razón"20.

Importa una vez más, por tanto, tener presente la perspectiva desde la cual un fenómeno o el mundo son calificados como "ilusiones" y, además, la arista semántica del término que Schopenhauer intenta destacar. Pues, tal como lo afirma Rábade,

El punto de arranque de una comprensión filosófica de la realidad ha de presentar para nuestro pensador [Schopenhauer] un carácter abierto, de suerte que se haga posible su puesta en cuestión y, a través de ello, su reinterpretación, así, el filósofo, al manejar los conceptos que le sirven de "material", ha de saber concederles en todo momento un cierto margen de problematicidad [...], que permita constantemente su revisión para adecuarse a la realidad intuitiva, integrando las nuevas facetas que eventualmente se descubren en ella ${ }^{21}$.

Podemos aventurarnos a presentar una gradación de la realidad y la ilusión desde las distintas perspectivas en la que se despliega el pensamiento schopenhaueriano. En primer lugar, la ilusión es una anormalidad en el modo habitual de la recepción empírica de las sensaciones que conduce a un fallo en la construcción del objeto de experiencia y de la intuición. Este fallo se produce dentro de la realidad fenomenal designada como "Wirklichkeit". En ese ámbito, lo real son las representaciones adecuadamente construidas por el entendimiento y es, desde esa posición, que las alucinaciones o anormalidades perceptivas pueden ser calificadas como "ilusorias". En segundo lugar, la realidad fenomenal (Wirklichkeit) en su conjunto y, con ello, el conocimiento científico en general, puede ser calificada como "ilusoria" justamente por ser una "apariencia" en tanto efecto (Wirkung) deducido a partir de las sensaciones. Esta forma de ilusión es la

${ }^{18}$ Cf. SCHOPENHAUER, A., MVR I, §6, p. 72-73.

${ }^{19}$ SCHOPENHAUER, A., MVR I, Crítica de la filosofía kantiana, p. 483.

20 SCHOPENAHUER, A., MVR I, §5, p. 63.

${ }^{21}$ RÁBADE OBRADO, A I. Conciencia y dolor. Schopenhauer y la crisis de la modernidad, p. 57.

Muerte, estética y filosofía: el arte de filosofar según Schopenhauer 
"ilusión trascendental"22. Aquí, lo propiamente ilusorio es la apariencia de las representaciones como realidades extra-mentales. Así, la ilusión se devela en el reconocimiento de la dependencia de la realidad fenomenal respecto del entendimiento, y de la naturaleza representacional del mundo. Este develamiento solo puede ser posible en un marco de referencia o grado distinto de realidad (Realität). Finalmente, el último grado de realidad es aquel donde la posibilidad de la ilusión ha sido disminuida o removida completamente. Aquí la Realität hace referencia a las especies naturales y la Voluntad, como trasunto del mundo fenoménico, lo representado en la representación.

Schopenhauer sostiene que, así como la ilusión es un engaño del entendimiento, el error (Irrthum) es un engaño de la razón ${ }^{23}$. Este tiene su origen en un defecto en la fundamentación de la verdad (Wahrheit).Schopenhauer afirma que, dado que tanto en el error como en la ilusión el engaño se debe a una deformación de la inferencia desde conclusión hacia el antecedente, "el error es, pues, totalmente análogo a la ilusión"24. Schopenhauer presenta tres tipos de errores: el primero es el error producido por una ilusión; el segundo, producido por una conexión causal posible que se toma por necesaria sin serlo; y el tercero, producido por una conexión causal imposible ${ }^{25}$. Ahora, el miedo a la muerte del hombre se explica, metafísicamente en la voluntad de vivir individual; pero, desde el punto de vista del fenómeno (el hombre concreto), este miedo tiene su origen en la razón misma en la medida en que el futuro y el pasado son abstracciones, cuyo dominio solo pueden ser $\operatorname{conceptos}^{26}$.Así, el miedo a la muerte se basa en una inferencia sobre la realidad del futuro. Dado que el futuro es una abstracción, es necesario aun rastrear cual sea su contenido intuitivo, sea este real o ilusorio. Schopenhauer sostendrá que aquel que se pregunta y angustia por la realidad de su pasado y por su porvenir, como si le fuera posible otro presente que el que efectivamente tiene,

ve su existencia y su tiempo como independientes entre sí y aquella como echada dentro de este: él supone en realidad dos ahoras, uno que pertenece al objeto y otro al sujeto (...). Pero en verdad [...] el presente lo

\footnotetext{
22 GÓMEZ FONTECHA, O. L. Ilusión, represión y liberación en Schopenhauer. Trabajo de grado para optar al título de Magistra en Filosofía, p. 12-13.

23 Cf. SCHOPENHAUER, A., MVR I, §6, p. 72.

${ }^{24}$ SCHOPENAHUER, A., MVR I, §15, p. 130.

25 Cf. Ibidem.

${ }^{26}$ Cf. SCHOPENHAUER, A., MVR I, §54, p. 334.

Muerte, estética y filosofía: el arte de filosofar según Schopenhauer
} 
constituye únicamente el punto de contacto del objeto, cuya forma es el tiempo, con el sujeto, que no tiene por forma ninguna de las figuras del principio de razón [...]. Solo el presente es lo que siempre existe y es definitivo. Siendo lo más efímero de todo cuando se lo concibe empíricamente, a la mirada metafísica, que pasa por alto las formas de la intuición empírica, se le presenta como lo único permanente ${ }^{27}$.

Importa aquí, una vez más, reconocer la perspectiva desde la cual se considera el tiempo: mientras que desde la mirada empírica el presente es efímero, desde la perspectiva metafísica es lo único realmente permanente. La experiencia empírica del tiempo debe ser considerada, en ese sentido, una ilusión. El carácter ilusorio de la sucesión temporal se refuerza aún más, si se reconoce que esta sucesión es la imperfección más importante de nuestro intelecto ${ }^{28}$. La realidad (Wirklichkeit) de la muerte y el temor a la misma, así entendidos, son errores que se basan en la ilusión de la sucesión temporal. Una de las tareas principales del pensador es, justamente, atacar el error $^{29}$. Así, es posible afirmar junto con Vasalou que la filosofía debe responder a un miedo fundado en un error. Este es producido por el giro objetivo sobre el curso temporal de la vida, el logro filosófico requiere el regreso sobre lo subjetivo y el redescubrimiento de la esencia del propio ser ${ }^{30}$.

Sostenemos, entonces, que la analogía que Schopenhauer establece entre la ilusión (Schein) y el error (Irrthum) puede ser extrapolada a la ilusión trascendental y el miedo a la muerte; en tanto ambos son genuinos problemas desde los que parte la filosofía. Aún más, la ilusión trascendental del tiempo es causa de la errónea inferencia de la muerte como fin definitivo del sujeto (no solo del individuo) y la angustia que con ello sobreviene. Con todo, la filosofía comienza justamente en el límite desde donde el error y la ilusión pueden ser reconocidos, lo que anticipa la posibilidad de su develamiento y disolución. La tesis idealista establece, al afirmar el carácter representacional del mundo, la dependencia de las representaciones respecto de algo más permanente y distinto que ellas: el sujeto. Esta primera toma de posición abre la posibilidad de una (re)descripción del mundo desde una perspectiva que permita develar la ilusión trascendental. De igual forma, el miedo a la muerte es un miedo respecto al presentimiento de una permanencia de la que no soy partícipe (valga decir

${ }^{27}$ SCHOPENHAUER, A., MVR I, §54, p. 335. El sombreado es mío.

${ }^{28} \mathrm{Cf}$. SCHOPENHAUER, A., MVR II, cap. 15, p. 173.

${ }^{29}$ Cf.SCHOPENHAUER, A.,MVR II, cap. 6, p. 98.

30 VASALOU, S. Schopenhauer and the Aesthetic Standpoint: Philosophy as a Practice of the Sublime, p. 45.

Muerte, estética y filosofía: el arte de filosofar según Schopenhauer 
"en tanto individuo"). La consideración de la finitud de la existencia deberá ser interpretada también desde el punto de vista de aquello que permanece. Para Schopenhauer, esto significará ascender en los distintos grados de conocimiento hasta el develamiento final de la Voluntad. Importa considerar, entonces, que para resarcir el error es posible el uso de argumentos y pruebas, pero estas dejan intactas a la ilusión 31 . Así, la filosofía deberá encontrar formas de intuición que permitan, al menos, alcanzar una "vista panorámica" de la naturaleza y la permanencia de las especies.

Al respecto, es interesante notar las facultades que Schopenhauer establece como requisitos del filosofar. De cara a la definición de filosofía, Schopenhauer reafirma que esta no puede agotarse en el análisis de conceptos, sino que el filósofo requierela capacidad de conectar conceptos e intuiciones. Esta no es meramente un producto del entendimiento y la razón, sino que requiere "según el grado de su perfección, la gracia, el juicio, la agudeza y el genio (Witz, Urtheilskraft, Scharfsinn, Genie). (...) El núcleo más íntimo de todo conocimiento auténtico y real es la intuición; y también cada nueva verdad es fruto de una de ellas. Todo pensamiento original se produce en imágenes; por eso la fantasía es un instrumento tan necesario para él (...)”32

Cada una de las facultades mencionadas por Schopenhauer merece un desarrollo en extenso. Por lo pronto, me contentaré con presentar la forma como el genio y la fantasía hacen posible el conocimiento filosófico. El genio es "la capacidad de conocer independientemente del principio de razón, es decir, en vez de las cosas individuales que tienen su existencia solo en la relación, conocer las ideas de las mismas, y así ser frente a ellas el correlato de la idea, es decir, no ya individuo sino puro sujeto del conocer." ${ }^{33}$. El conocimiento de la idea es central en el desarrollo de la filosofía de cara al conocimiento científico. Este último sistematiza y subsume particulares bajo conceptos generales que corresponden con los géneros y fuerzas con las que se explica científicamente la naturaleza. La filosofía busca comprender la experiencia en conjunto. Para ello, no basta el conocimiento de la fuerza vital que se ha identificado con la voluntad, sino que se debe comprender las formas generales en las cuales se objetiva la voluntad. Estas formas generales son, con propiedad, las ideas platónicas de la naturaleza orgánica, a través de las cuales se manifiesta la voluntad. Es decir, que aquello a lo que la ciencia solo puede

${ }^{31} \mathrm{Cf}$. SCHOPENHAUER, A., MVR I, §6, p. 73.

32 SCHOPENHAUER, A., MVR II, cáp. 7, p. 102.

${ }^{33}$ SCHOPENHAUER, A., MVR I, §37, p 248.

Muerte, estética y filosofía: el arte de filosofar según Schopenhauer 
acceder por abstracción de las relaciones entre los particulares se presenta en la experiencia estética como el objeto propio e inmediato de conocimiento. En la experiencia estética, la fantasía cumple un rol propiamente activo en el develamiento de las condiciones intelectuales y bilógicas que hacen posible la experiencia empírica. La intuición empírica, con propiedad, es meramente percepción y está siempre sujeta al espacio como condición de posibilidad. De ahí que la contemplación estética no esté sujeta a las mismas restricciones que la percepción empírica. Por el contrario, la intuición de las ideas platónicas cumple el rol de una bisagra que permite esclarecer momentáneamente la relación entre la voluntad y las representaciones. Así, la estética se formula, en parte, para "aclarar" (erläutern) la compresión expuesta "en abstracto" de la distinción entre inmanencia y trascendencia y para que "excepcionalmente [esta distinción] pueda presentarse también de forma intuitiva (intuitiveinstellen)”34. Frente a la experiencia estética, el sujeto se vuelve consciente de 1) que el mundo es propiamente una representación derivada de formas originales en las que la voluntad se objetiva y 2) que las representaciones no son ni pueden ser las cosas en sí, sino que suceden en el orden ontológico a un otro completamente distinto.

En la contemplación estética, afirma Schopenhauer, el sujeto se reconoce como "soporte del mundo (Träger der Welt)"35. Este reconocimiento solo es posible por la superación de la aversión interna a la tesis idealista mediante la contemplación de las formas originarias en relación con las cuales las condiciones que hacen posible la individualidad revelan su carácter ilusorio. Esta contemplación es una anormalidad de la naturaleza, en donde la voluntad pugna, a través del hombre, por volverse consciente de sí misma. La estética, así, configura para Schopenhauer una crítica de la racionalidad aparente de la experiencia empírica que devela, a la vez, la naturaleza íntima del mundo, las condiciones que hacen posible su objetivación (espacio, tiempo y causalidad) y las formas generales en las cuales la Voluntad se objetiva (las ideas platónicas).

A pesar de esto, las funciones del genio artístico y el filósofo son aun considerablemente distintas: en ambos se presenta el conocimiento del qué del mundo y de la vida, pero al filósofo le corresponde comunicarlo a través de conceptos adecuadamente constituidos a fin de no perder vinculación con su raíz intuitiva, y al genio artístico la comunicación a través de obras de arte que no son meras copias de la

${ }^{34}$ Cf. SCHOPENHAUER, A., MVR I, § 31, 227.

${ }^{35} \mathrm{Cf}$. SCHOPENHAUER, A., MVR I, § 34, 235.

Muerte, estética y filosofía: el arte de filosofar según Schopenhauer 
idea, sino que expresan afectiva y materialmente el proceso de objetivación de la voluntad en el mundo. El filosofar, como la filosofía misma, se encuentra en medio camino entre el arte y la ciencia. Por ello, el filósofo debe hacer uso de todas las facultades a su disposición: desde la razón y el juicio que permiten contrarrestar el error argumentativamente y subsumir los individuos bajo su respectivo concepto; hasta el genio y la fantasía que permiten el develamiento de la ilusión a través de una alteración de la intuición fundada en la transformación del propio sujeto.

¿Cómo se vence el miedo a la muerte a través de la vía estética? Tal como lo presentamos, el miedo a la muerte es abstracto, en la medida en que se basa en una inferencia errónea respecto a la realidad del futuro; desde la perspectiva metafísica tan solo el presente es real. Este presente ya no puede ser un presente temporal, sino, más bien la supresión del tiempo como condición a priori de las representaciones empíricas. Esta supresión es posible en la experiencia estética a través de la capacidad de genio que produce el conocimiento de la idea platónica. Esta condición es clave para la filosofía y su promesa de vencer el miedo a la muerte. Pues, si consideramos la vida filosóficamente,

\begin{abstract}
es decir, según sus ideas (Ideen), y encontraremos que ni la voluntad, la cosa en sí en todos los fenómenos, ni el sujeto del conocimiento, el espectador de todos ellos, son afectados por el nacimiento y la muerte. Nacimiento y muerte pertenecen al fenómeno de la voluntad, esto es, a la vida; y es esencial a esta presentarse en individuos que nacen y perecen en cuanto fenómenos pasajeros, surgidos en la forma del tiempo, de aquello que en sí mismo no conoce tiempo alguno pero que se ha de presentar justamente de la forma mencionada para objetivar su verdadero $\operatorname{ser}^{36}$.
\end{abstract}

Sin la experiencia estética y la facultad del genio, la consideración metafísica del mundo permanecería expuesta solo de forma abstracta y no brindaría ningún consuelo real ante la muerte. La experiencia estética permite que el sujeto que filosofa se reconozca como sujeto puro de conocimiento y portador del mundo, esto es, se reconoce como condición atemporal del devenir del mundo, que no se encuentra sometida a la muerte.

36 SCHOPENHAUER, A., MVR I, §54, 324.

Muerte, estética y filosofía: el arte de filosofar según Schopenhauer 


\section{Referencias bibliográficas}

\section{Primaria}

SCHOPENHAUER, Arthur. El mundo como voluntad y representación I. Traducción de Pilar López de Santa María. Madrid: Trotta, 2005.

SCHOPENHAUER, Arthur. El mundo como voluntad y representación II. Traducción de Pilar López de Santa María. Madrid: Trotta, 2005.

Secundaria

GÓMEZ FONTECHA, Olga Lucía. Ilusión, represión y liberación en Schopenhauer. Trabajo de grado para optar al título de Magistra en Filosofía. Bogotá: Pontificia Universidad Javeriana, 2015. $<$ https://repository.javeriana.edu.co/bitstream/handle/10554/17042/GomezFontechaOlgaLuc ia2015.pdf? sequence=1>. Consultado el 06/07/2017.

JACQUETTE, Dale. "Schopenhauer on Death". En: The Cambridge Companion to Schopenhauer, editadopor Christopher Janaway, Cambridge: Cambridge University Press, 2009.

RÁBADE OBRADO, Ana Isabel. Conciencia y dolor. Schopenhauer y la crisis de la modernidad. Madrid: Trotta, 1995.

SCHÖNDORF, Harald. "La muerte en la filosofía de Arthur Schopenhauer". En: Revista Portuguesa de Filosofía. Tomo 65: O Dom, a Verdade, e a Morte: Abordagens e Perspectivas, Braga: Universidade Católica Portuguesa, 2009, pp. 1193-1204.

VASALOU, Sophia. Schopenhauer and the Aesthetic Standpoint: Philosophy as a Practice of the Sublime, Cambridge: Cambridge university Press, 2013.

Recebido: 09/01/18

Received: 01/09/18

Aprovado: 20/01/18

Approved: 01/20/18 